Review

\title{
Evaluating right ventricular function to reveal cancer therapy cardiotoxicity
}

\author{
Alexey N. Sumin \\ Federal State Budgetary Scientific Institution "Research Institute for Complex Issues of Cardiovascular Diseases", Kemerovo, Russia
}

Received 30 October 2020, Revised 8 April 2021, Accepted 20 May 2021

(C) 2020, Russian Open Medical Journal

\begin{abstract}
Advances in cancer treatment resulted in augmented patient survival rate. However, anticancer therapy often causes heart damage in the form of a progressive systolic heart failure. Echocardiographic parameters of left ventricular function were conventionally used to detect early manifestations of cancer therapy cardiotoxicity. Improved diagnosing of the right ventricle condition revealed that it is negatively affected by chemotherapy as frequently as the left ventricle, and sometimes even earlier than the latter. Hence, currently, the right ventricle function and mechanics assessment techniques are actively developed for the chemotherapy cardiotoxicity diagnostic, primarily employing 3D echocardiography and speckle tracking analysis. The presented review provides relevant information on the matter and highlights insufficiently developed issues and fields of further research.
\end{abstract}

Keywords: cancer therapy cardiotoxicity, echocardiography, right ventricular.

Cite as Sumin AN. Evaluating right ventricular function to reveal cancer therapy cardiotoxicity. Russian Open Medical Journal $2021 ; 10$ : e0309.

Correspondence to Alexey N. Sumin. Address: Laboratory of Comorbidity in Cardiovascular Diseases, Federal State Budgetary Scientific Institution "Research Institute for Complex Issues of Cardiovascular Diseases”, 6 Sosnoviy Blvd, Kemerovo 650002, Russia, Phone: +7 (3842) 64-44-61, +7-903-940-86-68. Fax: +7 (3842) 64-27-18. E-mail: an sumin@mail.ru, sumian@kemcardio.ru.

\section{Introduction}

The personalized medicine, a.k.a. precision medicine, is the trend of recent years, involving various aspects of an individual approach to patients. Among others, such personalized approach includes studying comorbid conditions that may affect the therapy and prognosis for patients. One of such comorbid conditions is cardiovascular oncology. Essentially, significant progress in detection and treatment of oncological pathology has led to a substantially improved survival of such patients. On the other hand, the improvement in acute cardiac disease treatment is accompanied by an increase in the chronic cardiovascular patient numbers. As a result, the cohort of patients with a combination of oncological and cardiovascular diseases is growing [1]. Hence, currently, cardiovascular oncology studies heart tumors, tumor invasion detection in the heart tissue, as well as preoperative patient assessment in surgical oncology [2]. Nonetheless, major attention is paid to the cancer therapy cardiotoxicity - first of all, to the development of systolic dysfunction and heart failure. The research focus is upon the following issues: pathophysiological mechanisms of cardiotoxicity [3], primary prevention of cardiotoxicity in high-risk patients, monitoring of early manifestations of cardiotoxic effects, secondary cardiotoxicity prevention, as well as diagnosis and treatment of other cardiotoxic manifestations (coronary artery disease and myocardial ischemia, myocarditis, amyloidosis, pericardial disease, peripheral vascular disease, and vascular dysfunction) [4].

Strategies for cardiotoxicity screening and detection encompass cardiac imaging (echocardiography, radionuclide ventriculography, cardiovascular magnetic resonance imaging) and biomarker assessment (troponin, natriuretic peptides). The choice of methods depends on local experience and the availability of diagnostic procedures [5]. Echocardiography is most frequently used for detecting systolic dysfunction and heart failure. The conventional cardiotoxicity criteria include a decrease in left ventricular ejection fraction (LVEF) $>10 \%$ below baseline values, along with a relative (i.e., percentage) reduction in the left ventricular global longitudinal strain (LV GLS) $>15 \%$ below the baseline.

At the same time, early cardiotoxicity detection allows timely initiation of heart failure therapy, which significantly improves the prognosis for such patients $[5,6]$. That is why, it was proposed to additionally evaluate other echocardiography parameters to detect early cardiotoxic manifestations (such as diastolic dysfunction using tissue Doppler and strain imaging, 3D echocardiography, and the right heart assessment). This review is focused upon the right ventricle (RV) function evaluation in the course of developing cardiotoxicity caused by anticancer medicinal drugs.

\footnotetext{
Essential facts about studies evaluating RV function vs. cardiac toxicity diagnosing

Currently, RV dysfunction is recognized among the most important causes of cardiovascular pathophysiology. Besides, it is an independent predictor of adverse events and mortality in a wide range of ailments: heart failure, valvular pathology, pulmonary hypertension, coronary artery disease, and even arterial hypertension [7-9]. At present, a widespread opinion prevails that there are fewer RV compensatory reserves than those of the left ventricle, hence RV dysfunction may occur earlier.
} 
However, an accurate evaluation of the RV condition via conventional echocardiography remains challenging due to intrinsic limitations of this imaging technique, along with complex geometry and contraction mechanisms of the RV. Recent developments in echocardiography expand the possibilities of using the RV parameter assessment in condition of various pathologies [10].

The cardiotoxicity assessment is no exception, which can be deduced from the increased number of publications in the PubMed database yielded by the search using the keywords right ventricular and cardiotoxicity (Figure 1). The studies of the RV structure and function in patients with antineoplastic treatment disclose the conflicting results. For example, the review by Tadic et al. [11] included the data of ten studies involving a limited number of enrolled patients (9-60). Evaluation of the RV systolic function was undertaken in all ten published studies: against the background of chemotherapy, it declined in five cases, while it remained unchanged in other five. RV diastolic function was assessed in five studies; in the course of treatment, it declined in two cases, and remained unchanged in other three. Just three studies investigated the RV mechanics against the background of antineoplastic treatment, and in all cases, a decline in the right ventricular global longitudinal strain (RV GLS) was observed. Apparently, the RV shape, its geometry and position in the ribcage may be the causes of the difficulties in assessing its size and function.

In addition, the attention was drawn to various imaging techniques (echocardiography, radionuclide diagnostic, and magnetic resonance imaging), and also to the set of various indicators characterizing the RV systolic and diastolic functions. Accordingly, it was problematic to interpret the results of such studies (whether there was no impact on RV - or there was no effect on the selected indicator for assessing the RV function). For example, our experience in studying the RV diastolic function in pulmonary hypertension implied the necessity to study a set of indicators that could change in different ways with the disease progress [12]. At the same time, the conventional indicator (E/A ratio on the tricuspid valve) was most closely associated with the development of postoperative heart failure in coronary artery bypass grafting, while other parameters of RV diastolic function were not [13].
The newest reviews highlighted the expediency of using recently developed imaging techniques, such as 3D echocardiography and speckle tracking analysis in diagnosing RV dysfunction. The combined results obtained via various echocardiographic methods allow obtaining a deeper understanding of the RV pathology, which, in our opinion, would lead to a more accurate diagnosis and better clinical management of patients with cardiovascular diseases in general [10], as well as during their anticancer therapy [14].

A recently published review [14] was devoted to studies on using the RV myocardial deformation to detect cancer therapy cardiotoxicity. It included the data from eight research projects undertaken in 2015-2019. Indeed, all these studies showed a decrease in the RV movement mechanics in conditions of the antineoplastic treatment cardiotoxicity development. Possible clinical significance of RV (vs. LV) function assessment was emphasized. The study by Chang WT et al [15] demonstrated that the value of the RV free wall longitudinal strain after the first course of epirubicin in patients with breast cancer was significantly reduced and was associated with the onset of dyspnea symptoms, which was not observed for other echocardiographic parameters. The independent prognostic value of this parameter was also shown in patients with non-small cell lung cancer of stage III during the ongoing chemo-radiation therapy; the worst prognosis was in patients with a decrease of $\geq 10.1 \%$ from the baseline value prior to the therapy [16]. Nevertheless, we conclude with some reservation that it is advisable to study the RV strain parameters in cancer patients to assess the cancer therapy cardiotoxicity [14]. A more definitive conclusion is difficult to draw, since most studies included in our review involved the methods that were more informative for RV function assessment versus the LV function evaluation. Therefore, a comparison of diagnostic efficacy, if any, could not be considered correct.

It is clear that current ongoing studies should close this evidential gap: if RV changes occur earlier than in the LV, this would allow detecting cardiotoxicity at the earliest stage of its development. Early diagnostic of cardiotoxicity may have a beneficial effect on the patient's prognosis, thus emphasizing the significance of studying the RV function.

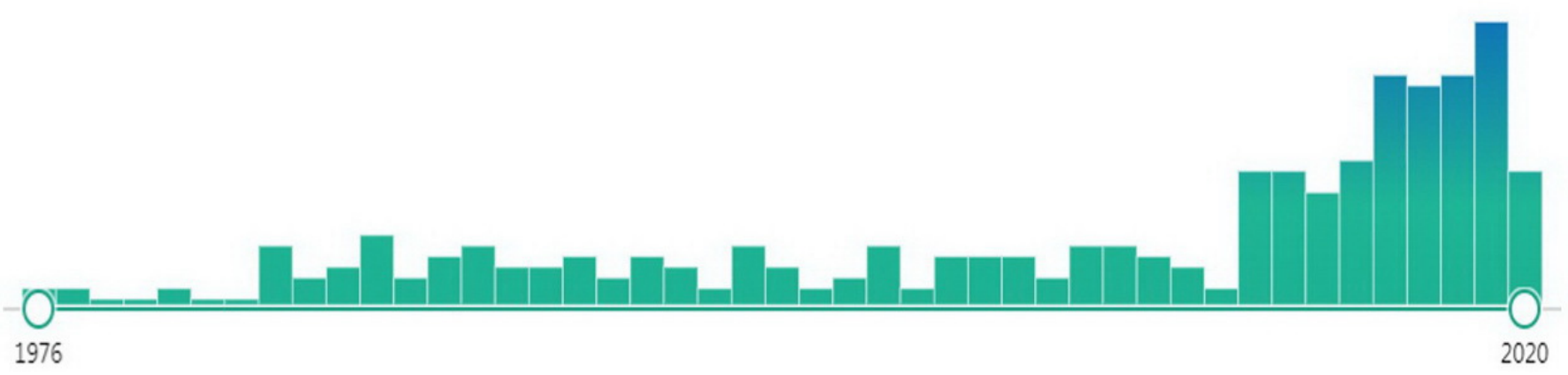

Figure 1. Number of publications in the PubMed database yielded by the 'right ventricular' and 'cardiotoxicity' queries 


\section{Recent studies on RV function in detecting cardiotoxicity}

Recent published sources provided additional information on this issue. Cherata DA et al. [17], in the course of a six-month follow-up treatment of 68 patients with anticancer therapy, established that cardiotoxicity manifestations have developed in 20 patients. Ninety percent of patients from this group received anthracycline-based treatment: doxorubicin and cyclophosphamide, followed by bleomycin or vincristine and monoclonal antibody rituximab: R-CHOP, R-FCM, R-BEACOP regimens, whereas just $10 \%$ of patients were treated with bortezomib. In this study, in the whole group of examined patients, there was a statistically significant $(p<0.0001)$ decline in the values of three parameters (LVEF, left ventricular LGS, and free wall strain of the RV). At the same time, in patients with cardiotoxicity development, a decline in LV indicator values was more pronounced than in the RV parameters $(p<0.0001$ and $p=0.005$, respectively). Hence, the authors of the article proposed to evaluate the RV longitudinal strain to identify its subclinical lesion in conditions of LV dysfunction development, associated with antineoplastic treatment. It is difficult to say how useful this recommendation is for clinical practice, because it is entirely unclear how such subclinical RV dysfunction could somehow affect the treatment tactics or prognosis in case of obvious LV dysfunction.

In this regard, the outcomes of the study by Planek et al. are more interesting [18]. They examined 35 adult lymphoma patients treated with doxorubicin. Prior to the therapy, all patients had a normal LVEF and no cardiovascular pathology. When re-examined six months later, the entire examined patient cohort had slightly reduced LVEF (63.3 $\pm 6.2 \%$ versus $61.6 \pm 11.1 \%, p=0.374)$, while studied RV indicators have changed for worse. E.g., the authors observed the reduction in RV FAC from $47.3 \pm 4.4 \%$ to $43.7 \pm 3.9 \%$; in RV FWS from $-24.9 \pm 3.3$ to $-22,2 \pm 2.9$; and in RV GLS from $-22.4 \pm 4.1$ to $-20.6 \pm 3.4$ (in all cases, $p<0.01$ ). Besides, it was established that the RV function reduction occurred exclusively in patients with a cumulative dose of doxorubicin exceeding $200 \mathrm{mg} / \mathrm{m}^{2}$ [18]. Despite the persuasiveness of the data, it is noteworthy that while the authors used sufficiently modern and informative assessment methods for the RV, including myocardial strain investigation, the left ventricle was evaluated solely via monitoring of its ejection fraction, which undoubtedly represented the limitation of this study.

In another recent study, Zhao $R$ et al. [19] investigated the dynamics of 3D echocardiography in 74 patients with diffuse large B-cell lymphoma who received six courses of anthracycline-based treatment (R-CHOP therapy). This research is interesting for several reasons. First, the authors used indicators of the right (rather than left) ventricle as cardiotoxicity criteria: a relative reduction in the $\mathrm{RV}$ ejection fraction $>10 \%$, or its relative reduction $>5 \%$ given the absolute values $<45 \%$. Second, in order to reveal early cardiotoxicity signs, they compared the parameters of both ventricles, which could help in the future selecting the optimal set of signs implying the occurrence of cardiotoxicity. By the end of the fourth course, the authors observed an increase in enddiastolic and end-systolic volumes of the RV, along with a decline in the RV longitudinal free wall strain in 3D mode and in LV GLS (for all cases, $p<0.05$ ). Upon completion of six therapeutic courses, the cardiotoxicity (according to the RV criteria) has developed in 27 patients, while no reduction in LV systolic function was observed. Among the studied parameters after four therapeutic courses with subsequent cardiotoxicity development, only two indicators of RV function were independently associated: the dynamics of the RV end-systolic volume and a decline in the RV longitudinal free wall strain in 3D mode (both compared with their initial values) [19]. One could say, of course, that the LV parameters did not work due to the fact that reduction in right ventricular ejection fraction was the cardiotoxicity criterion here. Indeed, there was no significant reduction detected in the LVEF (only by $5 \%$, while the guidelines recommend over $10 \%$ [5]), and the authors did not assess cardiotoxicity via some other LV parameter. As for the latter, the authors did not evaluate its GLS, even though, according to their data, the reduction in this indicator after six courses has reached a significant level (by $21 \%$, whereas the guidelines recommend over 15\% [5]).

Since an assessment of LV longitudinal strain is considered more sensitive cardiotoxicity sign than LVEF, this fact implies the study limitation. Whether RV indicators are more sensitive in this regard is still unclear, especially since there are virtually no established diagnostic RV criteria for cardiotoxicity. However, there is a theoretical background for employing RV parameters to evaluate the chemotherapy cardiotoxicity. For example, there is some evidence that anthracycline therapy primarily affects the subendocardial layers of the LV, while the preserved function of subepicardial layers allows maintaining the LV pumping function for relatively long time [20]. Since the RV wall is significantly thinner, its pumping function decompensation occurs earlier than in the LV [19]. At the same time, the use of 2D speckle tracking technology, permitting separate assessment of deformation in the subendocardial vs. subepicardial layers, could also solve the problem of identifying subclinical signs of cardiotoxicity in LV assessment [20]

It seems that the data of Zhao R. et al. [19] have benefited so far to the most significant progress in substantiating the need of assessing the RV function for identifying cardiotoxic manifestations of antineoplastic therapy. However, certain limitations of this study could not be ignored: lack of comparison between the 3D echocardiography data versus cardiac MRI or computed tomography data, relatively small sample size and a short followup period. Likewise, it is impossible to completely exclude the effect of preload changes on such RV preload-dependent parameters as its volume and strain [19]. Further studies undertaken on large samples and with a longer follow-up period are required to confirm the results of this research, as well as to develop standardized clinically significant criteria for cardiotoxicity developed in the RV [21].

In our review, we did not consider the potential cellular mechanisms of RV remodeling during chemotherapy, since they have already been discussed in detail in previous reviews by international and Russian authors $[3,11]$. Other existing methods for assessing RV function during the chemotherapy (MRI, radionuclide ventriculography, positron emission and computed tomography) were not considered as well. The fact is that they have already been discussed in earlier reviews [4, 11]. Besides, these methods are still less available in actual clinical practice.

However, the routine use echocardiographic indicators of the RV function could also encounter certain difficulties due to sonographer inertia. In this regard, the study by Schneider $\mathrm{M}$ et al. is very indicative [22]: the authors have evaluated the current use of clinical standards for RV function quantification in a clinical practice. Because many parameters are time-consuming and 
require specific computer appliances, most of RV markers are not widely used. The total of 1150 participants from 109 countries participated in the survey. They were asked to indicate the RV parameters (visual assessment, tricuspid annular plane systolic excursion - TAPSE, $s$ ' in tissue Doppler mode, fractional area change, myocardial performance index, 3D echocardiography, $\mathrm{dp} / \mathrm{dt}$, longitudinal strain), which they have actually used in their clinical practice. Despite existing guidelines on necessity to use at least one quantitative marker in addition to visual grading in the assessment of RV function [23], 39\% of the participants used only one method in their daily clinical practice, $35 \%$ used two methods, and $26 \%$ used three or more methods. Visual assessment was used as the only parameter by $23 \%$ of participating sonographers [22]. Such approach cannot be considered acceptable, which was confirmed by the subsequent research conducted by the same authors. In an anonymous test via Internet, sonographers were assessing RV systolic function in 10 patients with varying dysfunction degrees using purely visual RV assessment. Two skills were evaluated: (1) the ability to discriminate between normal and reduced RV systolic function; and (2) the ability to identify the correct degree of RV systolic dysfunction (against the background of MRI data). For detecting reduced RV systolic function, sensitivity ranged from $95.8 \%$ to $97.1 \%$, depending on a participant's experience, while specificity ranged from $42.5 \%$ to $55.7 \%$. When determining the degree of RV dysfunction, even experienced physicians came up with a diagnosis that did not match MRI findings in $>40 \%$ of cases. This finding implied that visual evaluation should be combined with the assessment of other RV echocardiographic parameters [24]. According to the abovementioned survey, the most frequently used techniques included visual assessment (72\% of cases), TAPSE $(69 \%)$, and $s^{\prime}$ in tissue Doppler imaging (31\%). What is especially distressing, an assessment of longitudinal strain and 3D echocardiography were rarely used, regardless of the region and economic factors ( $3 \%$ and $1 \%$ of cases, respectively) [22]. Moreover, nearly all recent studies emphasized the lack of information content in traditionally used indicators of RV systolic and diastolic functions in identifying RV cardiotoxicity, especially at the early stages $[11,17-19]$.

\section{Conclusion}

In the cancer therapy cardiotoxicity diagnosis, the main trend over recent years involved using contemporary echocardiographic research methods (3D echocardiography and assessment of myocardial strain). First of all, they were developed for the detailed assessment of the LV function and mechanics. However, the improvement of diagnostic procedures has also affected the right ventricle investigation techniques: the obtained results implied that its damage due to chemotherapy cardiotoxic effects is simultaneous with the LV damage, and may even precede it.

Accordingly, adequate assessment of RV function and mechanics should be a mandatory component of each echocardiographic study, since solely visual assessment of RV function is obviously insufficient. To monitor cardiotoxic effects on the RV myocardium, it is desirable to use indicators of its mechanics and 3D echocardiography data. To confirm the possibility of RV assessment for early detection of subclinical cardiotoxicity manifestations, clarification of their prognostic value, and development of standardized criteria for cardiotoxic RV damage detection, further larger (and, preferably, multicenter) studies are required.

\section{Conflicts of interest}

The author declares no conflict of interest.

\section{References}

1. Cubbon RM, Lyon AR. Cardio-oncology: Concepts and practice. Indian Heart J 2016; 68 Suppl 1(Suppl 1): S77-85. https://doi.org/10.1016/j.ihj.2016.01.022.

2. Sumin AN. Preoperative management of patients with cardiovascular diseases for elective cancer surgery. Complex Issues of Cardiovascular Diseases 2019; 8(1): 123-133. Russian. https://doi.org/10.17802/23061278-2019-8-1-123-133.

3. Chaulin AM, Abashina OE, Duplyakov DV. Pathophysiological mechanisms of cardiotoxicity in chemotherapeutic agents. Russ Open Med J 2020; 9: e0305. https://doi.org/10.15275/rusomj.2020.0305.

4. Biersmith MA, Tong MS, Guha A, Simonetti OP, Addison D. Multimodality cardiac imaging in the era of emerging cancer therapies. J Am Heart Assoc 2020; 9(2): e013755. https://doi.org/10.1161/jaha.119.013755.

5. Zamorano JL, Lancellotti $P$, Rodriguez Muñoz D, Aboyans V, Asteggiano R, Galderisi M, et al.; ESC Scientific Document Group. 2016 ESC Position Paper on cancer treatments and cardiovascular toxicity developed under the auspices of the ESC Committee for Practice Guidelines: The Task Force for cancer treatments and cardiovascular toxicity of the European Society of Cardiology (ESC). Eur Heart J 2016; 37(36): 2768-2801. https://doi.org/10.1093/eurhearti/ehw211.

6. Lenneman CG, Sawyer DB. Cardio-Oncology: An Update on Cardiotoxicity of Cancer-Related Treatment. Circ Res 2016; 118(6): 1008-1020. https://doi.org/10.1161/circresaha.115.303633.

7. Haddad F, Doyle R, Murphy DJ, Hunt SA. Right ventricular function in cardiovascular disease, part II: Pathophysiology, clinical importance, and management of right ventricular failure. Circulation 2008; 117(13): 1717-1731. https://doi.org/10.1161/circulationaha.107.653584.

8. Tadic M, Cuspidi C, Bombelli M, Grassi G. Right heart remodeling induced by arterial hypertension: Could strain assessment be helpful? J Clin Hypertens (Greenwich) 2018; 20(2): 400-407. https://doi.org/10.1111/ich.13186.

9. Surkova E, Muraru D, Genovese D, Aruta P, Palermo C, Badano LP. Relative prognostic importance of left and right ventricular ejection fraction in patients with cardiac diseases. J Am Soc Echocardiogr 2019; 32(11): 1407-1415. https://doi.org/10.1016/i.echo.2019.06.009.

10. Surkova E, Kovács A. Comprehensive echocardiographic assessment of the right ventricular performance: Beyond TAPSE and fractional area change. Russian Journal of Cardiology 2020; 25(S3): 4067. https://doi.org/10.15829/1560-4071-2020-4067.

11. Tadic $M$, Cuspidi $C$, Hering $D$, Venneri L, Danylenko $O$. The influence of chemotherapy on the right ventricle: did we forget something? Clin Cardiol 2017; 40(7): 437-443. https://doi.org/10.1002/clc.22672.

12. Sumin AN., Arhipov OG. Markers of right ventricular diastolic dysfunction in patients with pulmonary hypertension. Clinical Medicine 2018; 96(1): 30-37. Russian. https://doi.org/10.18821/00232149-2018-96-1-30-37.

13. Sumin AN, Korok EV, Sergeeva TJ. Preexisting right ventricular diastolic dysfunction and postoperative cardiac complications in patients undergoing nonemergency coronary artery bypass surgery. J Cardiothorac Vasc Anesth 2021; 35(3): 799-806. https://doi.org/10.1053/i.jvca.2020.09.100.

14. Keramida K, Farmakis D. Right ventricular involvement in cancer therapy-related cardiotoxicity: The emerging role of strain echocardiography. Heart Fail Rev 2020; 26(5): 1189-1193. https://doi.org/10.1007/s10741-020-09938-8.

15. Chang WT, Shih JY, Feng YH, Chiang CY, Kuo YH, Chen WY, et al. The early predictive value of right ventricular strain in epirubicin-induced cardiotoxicity in patients with breast cancer. Acta Cardiol Sin 2016; 32(5): 550-559. https://doi.org/10.6515/acs20151023a. 
16. Chen L, Huang J, Wu W, Ta S, Xie X. The impact of right ventricular function on prognosis in patients with stage III non-small cell lung cancer after concurrent chemoradiotherapy. Int J Cardiovasc Imaging 2019; 35(6): 1009-1017. https://doi.org/10.1007/s10554-019-01590-0.

17. Cherata DA, Donoiu I, Diaconu R, Glodeanu A, Cârstea D, Militaru C, et al. Longitudinal strain analysis allows the identification of subclinical deterioration of right ventricular function in patients with cancer therapy-related left ventricular dysfunction. Discoveries (Craiova) 2019; 7(2): e94. https://doi.org/10.15190/d.2019.7.

18. Planek MIC, Manshad A, Hein K, Hemu M, Ballout F, Varandani R, et al. Prediction of doxorubicin cardiotoxicity by early detection of subclinical right ventricular dysfunction. Cardiooncology 2020; 6: 10. https://doi.org/10.1186/s40959-020-00066-8.

19. Zhao R, Shu F, Zhang $C$, Song F, Xu Y, Guo $Y$, et al. Early detection and prediction of anthracycline-induced right ventricular cardiotoxicity by 3-dimensional echocardiography. JACC CardioOncol 2020; 2(1): 13-22. https://doi.org/10.1016/i.jaccao.2020.01.007.

20. Kang $\mathrm{Y}$, Xiao $\mathrm{F}$, Chen $\mathrm{H}$, Wang $\mathrm{W}$, Shen $\mathrm{L}$, Zhao $\mathrm{H}$, et al. Subclinical anthracycline-induced cardiotoxicity in the long-term follow-up of lymphoma survivors: A multi-layer speckle tracking analysis. Arq Bras Cardiol 2018; 110(3): 219-228. https://doi.org/10.5935/abc.20180042.

21. Liu JE. Anthracycline-induced cardiotoxicity: remembering the forgotten ventricle. JACC CardioOncol 2020; 2(1): 23-25. https://doi.org/10.1016/i.jaccao.2020.02.012.

22. Schneider M, Aschauer S, Mascherbauer J, Ran H, Binder C, Lang I, et al. Echocardiographic assessment of right ventricular function: Current clinical practice. Int J Cardiovasc Imaging 2019; 35(1): 49-56. https://doi.org/10.1007/s10554-018-1428-8.

23. Rudski LG, Lai WW, Afilalo J, Hua L, Handschumacher MD, Chandrasekaran K, et al. Guidelines for the echocardiographic assessment of the right heart in adults: A report from the American Society of Echocardiography endorsed by the European Association of Echocardiography, a registered branch of the European Society of Cardiology, and the Canadian Society of Echocardiography. J Am Soc Echocardiogr 2010; 23(7): 685-713. https://doi.org/10.1016/j.echo.2010.05.010.

24. Schneider M, Ran H, Aschauer S, Binder C, Mascherbauer J, Lang I, et al. Visual assessment of right ventricular function by echocardiography: How good are we? Int I Cardiovasc Imaging 2019; 35(11): 2001-2008. https://doi.org/10.1007/s10554-019-01653-2.

\section{Authors:}

Alexey N. Sumin - MD, DSc, Head of the Laboratory of Comorbidity in Cardiovascular Diseases, Federal State Budgetary Scientific Institution

"Research Institute for Complex Issues of Cardiovascular Diseases", Kemerovo, Russia. http://orcid.org/0000-0002-0963-4793. 